\title{
Low Temperature Electrical Transport Studies of the Conducting Polymer Versicon ${ }^{\mathrm{TM}}$
}

\author{
Peter K. LeMaire \\ Department of Physics \& Engineering Physics, Central Connecticut State University, New Britain, CT, USA \\ Email: lemaire@ccsu.edu
}

How to cite this paper: LeMaire, P.K. (2019) Low Temperature Electrical Transport Studies of the Conducting Polymer Versicon $^{\mathrm{TM}}$. American Journal of Analytical Chemistry, 10, 504-512. https://doi.org/10.4236/ajac.2019.1010036

Received: September 17, 2019

Accepted: October 27, 2019

Published: October 30, 2019

Copyright ( 2019 by author(s) and Scientific Research Publishing Inc. This work is licensed under the Creative Commons Attribution International License (CC BY 4.0).

http://creativecommons.org/licenses/by/4.0/

\section{cc) (i) Open Access}

\begin{abstract}
Thermal analysis and low temperature D.C. electrical transport measurements of the conducting polymer Versicon ${ }^{\mathrm{TM}}$ were carried out between $20 \mathrm{~K}$ and $300 \mathrm{~K}$. The material was found to be stable up to $498 \mathrm{~K}\left(225^{\circ} \mathrm{C}\right)$, with a glass transition at $210 \mathrm{~K}\left(-63^{\circ} \mathrm{C}\right)$, and a crystalline transition at $436 \mathrm{~K}$ $\left(163^{\circ} \mathrm{C}\right)$. The electrical resistivity data best fitted the Fluctuation Induced Tunneling (FIT) model, suggesting that at low temperatures, the electron transport is by tunneling through thermally modulated barriers. The high temperature data best fitted the thermally activated hopping model, with an activation energy of $0.015 \mathrm{eV}$, suggesting that the thermally activated hopping may be a parallel transport process to fluctuation induced tunneling, becoming dominant at higher temperatures. From the FIT model the inter-particle distance was estimated to be $12 \AA$. The electrical transport results were also consistent with the assertion that Versicon ${ }^{\mathrm{TM}}$ forms spherical aggregates, creating conducting pathways even in an insulating matrix.
\end{abstract}

\section{Keywords}

Polyaniline, Electrical Transport, Thermal Analysis, Conducting Polymer, Low Temperature

\section{Introduction}

Polymers have found numerous technological applications, such as in automobile parts, sports equipment, electrical insulation, windshields for fighter jets, adhesives, paints and wood substitutes for construction among others. Until the Nobel Prize winning work of Alan J. Heeger, Alan G. MacDiarmid, and Hideki Shirakawa, polymers were synonymous with insulators. The need for more versatile and lightweight conductors for use in rapidly changing technologies, especially in miniature applications, fueled the drive to look to polymers for 
answers. Polymers are lightweight, easily cast into films and thus have the requisite properties for the numerous miniature and lightweight applications in practically all areas of current technology from the health fields [1] to communications. The numerous practical applications have brought about a truly multidisciplinary approach involving Physicists, Chemists, Materials Scientists and Engineers to the study and development of polymers, and to discover and synthesize electrically active polymers for a wide range of applications including energy storage systems (batteries and fuel cells), piezoelectric and pyroelectric applications such as actuators, and a wide range of sensors [2] [3]. Conducting polymers have also found uses in anti-static or electromagnetic shielding applications in the form of paints. Research continues in the study of conducting polymers for use in aircrafts for shielding, energy dissipation, and corrosion resistance applications [4] [5]. For example, wiring on airplanes uses metals which have much higher mass densities than conducting polymers. Thus, if eventually airplanes and other transportation vehicles are wired using conducting polymers, the total payload would be drastically reduced leading to subsequent significant savings in fuel and cost. These efforts have led to a large number of electrically active polymers.

Due to the linear nature of polymers, electrical conductivity in polymers, which takes place as a result of doping a polymer backbone, is often non-isotropic [6]. Thus, processing of conducting polymers, in the form of powders for use in various applications, leads to a reduction in the optimum conductivity. The problem is exacerbated when such conducting polymers are used as fillers in preparing conducting blends, as there is often non-uniform conductivity throughout the blend. One of the most promising polymers for electroactive applications is polyaniline (PANI) [7]. A number of years ago, Allied Signal reported a conducting polymer composed of submicron particles with a bulk conductivity of about $2-4 \mathrm{ohm}^{-1} \cdot \mathrm{cm}^{-1}$ and specific gravity of 1.36 . This conducting polymer originally with the trade name Versicon ${ }^{\mathrm{TM}}$, is a polyaniline (PANI) doped with a proprietary organic sulfonic acid [7]. It was reported to have a tendency to self aggregate in blends, forming uniform conductive pathways in an otherwise insulating matrix [8].

Conducting polymers are not just great for their numerous technological applications, they also make very interesting Physics and Chemistry. Most applications of conducting polymers involve some level of processing which in turn may affect their properties. In line with the original assertion by Allied Signal [7], Versicon was confirmed to self aggregate in an insulating matrix of PolyVinylChloride (PVC) [9]. The Versicon in PVC work suggested that the Versicon paricles in PVC were touching or almost touching particles, and also at low temperatures, the I-V curves were non-ohmic. The latter was attributed to high field effects, presumably brought about by the presence of the insulating PVC layers, albeit very thin, between aggregates of Versicon. In the current work, the electrical transport properties of pure Versicon in the form of pressed pellets, 
were studied to ascertain the validity of latter observations found in the Versicon-PVC blend, and provide more clarity and fundamental understanding of the mode of transport of these conducting polymers in blends.

\section{Experimental}

Although most applications of conducting polymers are at ambient temperatures, the electrical transport studies in our laboratory were done at low temperatures, since at low temperatures, thermal noise is reduced to allow for better understanding of the mode of transport in these materials. Since thermal noise is a temperature dependent signal which increases with temperature, low temperatures improve signal to noise ratio in measurements, leading to clearer observation of underlying phenomena. Pellets of powdered Versicon were pressed using a standard lab press at various pressures from 4 psi to $10 \mathrm{psi}$, and at about $100^{\circ} \mathrm{C}$. The pellets were heated to reduce the effect of adsorbed moisture in the Versicon powder and also to reduce trapped air in the samples during the pressing process. The pressed pellets resulted in slightly varying densities between 1.25 $\mathrm{g} / \mathrm{cm}^{3}$ to $1.32 \mathrm{~g} / \mathrm{cm}^{3}$. A representative sample was thus used in this experiment. The pellets were cut into rectangular shapes for density and resistivity measurements. Thermogravimetric Analysis (TGA) and Differential Scanning Calorimetry (DSC) of the samples were done between $-130^{\circ} \mathrm{C}$ and $300^{\circ} \mathrm{C}$. Resistivity measurements were carried out, using a Direct Current (D.C.) four lead method in the temperature range of $20 \mathrm{~K}$ to $300 \mathrm{~K}$. Voltage vs. current data were collected at each temperature using a Keithley ${ }^{\mathrm{TM}}$ constant current source, a Keithley ${ }^{\mathrm{TM}}$ digital multimeter, a LakeShore ${ }^{\mathrm{TM}}$ temperature controller, and a CTI-Cryogenics closed cycle refrigerator. The data acquisition was done via a computer-controlled data acquisition system using a home written data acquisition routine.

\section{Results and Analysis}

\subsection{Thermal Analysis}

The TGA measurements (Figure 1) showed small mass losses up to about $100^{\circ} \mathrm{C}$ and no losses till about $225^{\circ} \mathrm{C}$. The initial mass loss may be due to adsorbed moisture, and the precipitous and significant (over 30\%) mass losses after $225^{\circ} \mathrm{C}$ indicates decomposition of the sample [10]. This suggests that Versicon is stable up to $225^{\circ} \mathrm{C}$.

From the DSC measurements (Figure 2), it can seen that there is a phase transition at about $-63^{\circ} \mathrm{C}(210 \mathrm{~K})$, which we believe is a glass transition $\left(T_{g}\right)$ [11] suggesting that Versicon is in a rubbery state above this temperature and goes into a glassy state below this temperature. This low $T_{g}$ supports Versicon's commercial use in conductive paints and other ambient applications where the rubbery state of conductive polymers is desirable. Another transition was observed at about $163^{\circ} \mathrm{C}$, a possible crystalline transition $\left(T_{c}\right)$, as seen from Figure 3. 


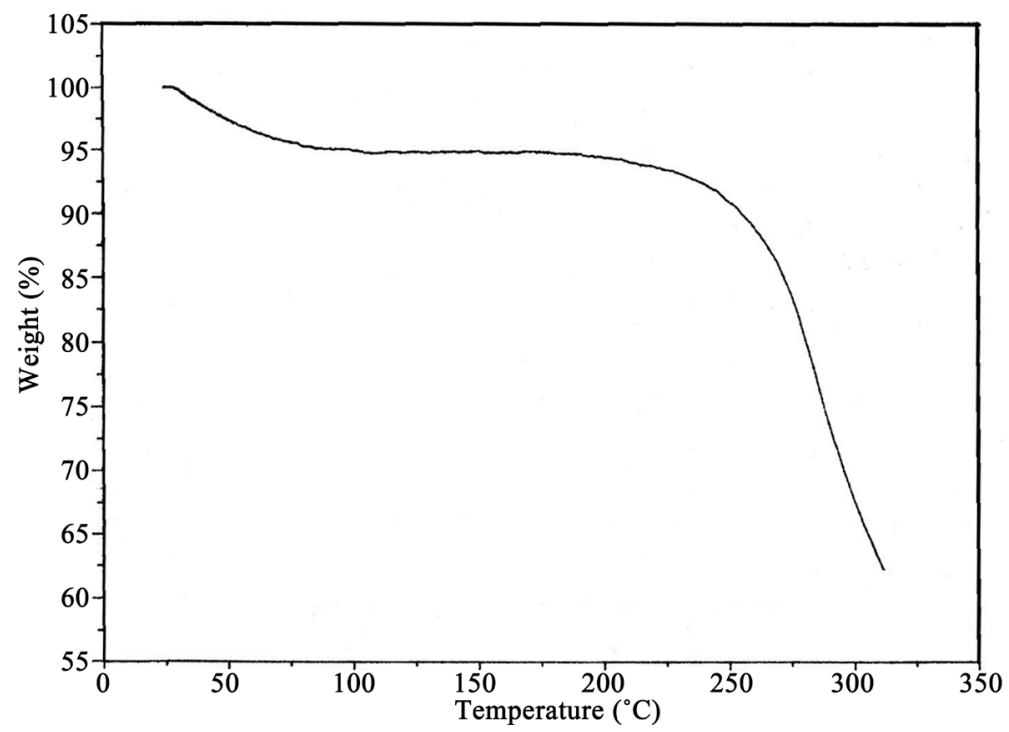

Figure 1. Thermogravimetric (TGA) Analysis of Versicon ${ }^{\mathrm{TM}}$ between $25^{\circ} \mathrm{C}$ and $300^{\circ} \mathrm{C}$.

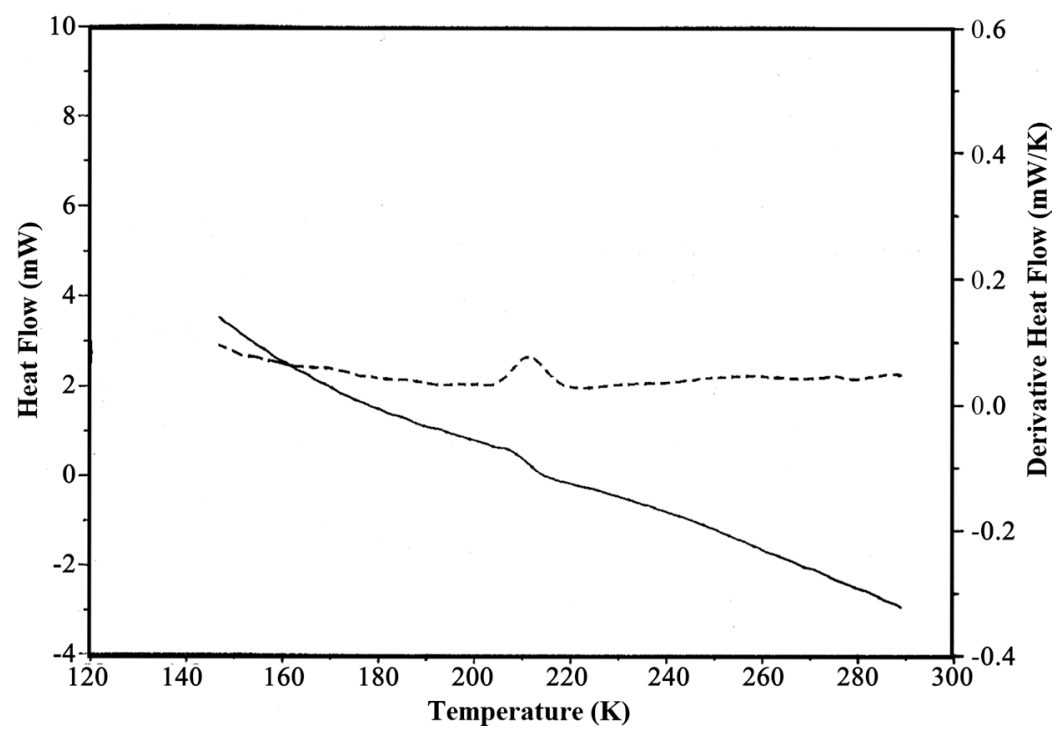

Figure 2. Differential Scanning Calorimetry (DSC) of Versicon ${ }^{\mathrm{TM}}$ between $150 \mathrm{~K}$ $\left(-123^{\circ} \mathrm{C}\right)$ and $290 \mathrm{~K}\left(17^{\circ} \mathrm{C}\right)$, showing a glass transition at about $210 \mathrm{~K}\left(-63^{\circ} \mathrm{C}\right)$. The continuous line is the heat flow and the dotted line is the derivative of the heat flow.

\subsection{Electrical Transport}

Conducting polymers have been known to have a number of conducting mechanisms and the mobile particles may be ions and/or electrons. The resistivity data were analyzed using known electrical transport models. For ionic transport, D.C. conductivity is non-ohmic, and the mode of transport may be by hopping from site to site in the polymer host, or through a liquid-like motion [12]. The hopping mode of ionic transport leads to a conductivity as a function of temperature which is of the Arrhenius form given by $\sigma T=A \exp (-E / k T)$, where $E$ is the activation energy, and $T$ is the temperature. The liquid-like mode of ionic transport results in a conductivity vs. temperature function given by 
$\sigma T=A \exp \left(-B /\left(T-T_{o}\right)\right)$. In an earlier work, Versicon blended in PVC was studied in a similar temperature range as this work, and the electrical transport data showed non-linear I-V characteristics at temperatures below $50 \mathrm{~K}$ [9]. In contrast, the electrical resistivity data, was ohmic over the entire temperature range, including the low temperature range, as seen in Figure 4, ruling out ionic mobility as

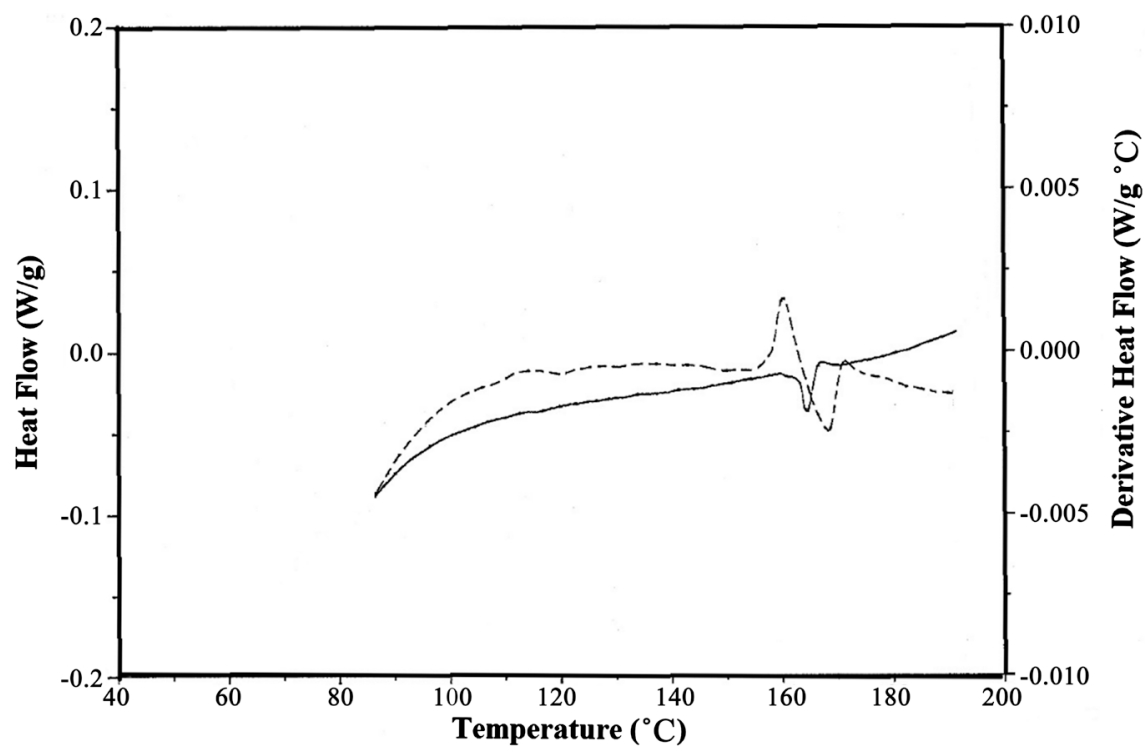

Figure 3. Differential Scanning Calorimetry (DSC) of Versicon ${ }^{\mathrm{TM}}$ between $90^{\circ} \mathrm{C}$ and $190^{\circ} \mathrm{C}$, showing a crystalline transition at about $163^{\circ} \mathrm{C}$. The continuous line is the heat flow and the dotted line is the derivative of the heat flow.

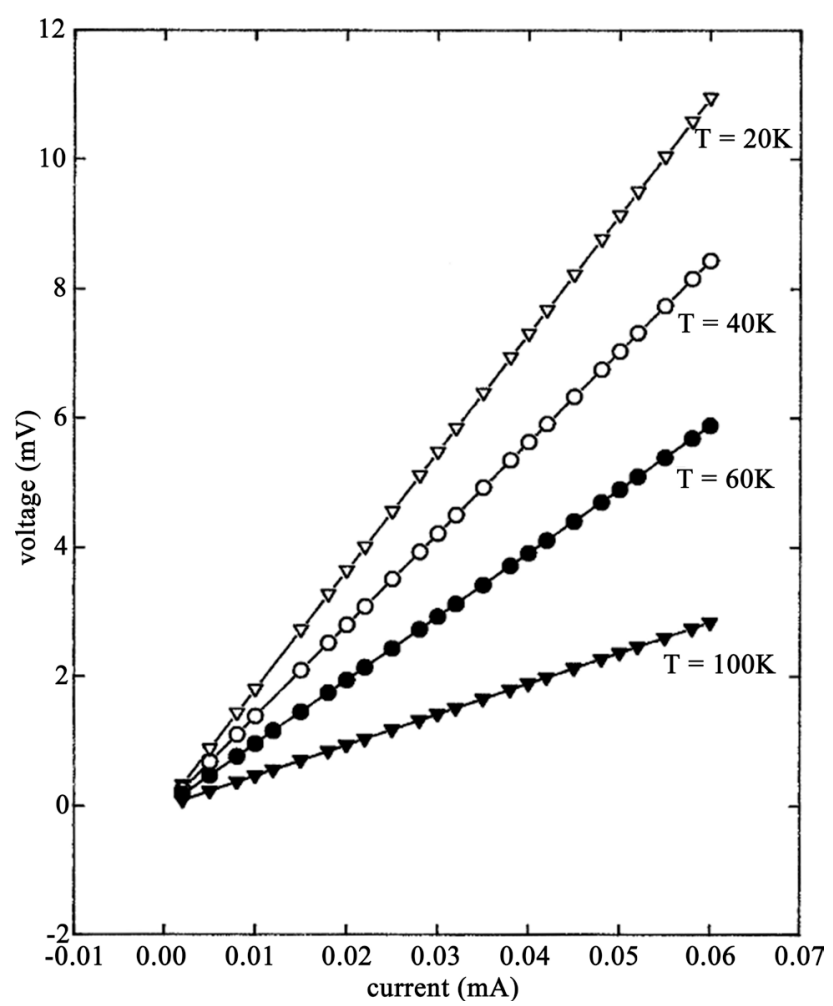

Figure 4. Voltage vs. Current data at and below $100 \mathrm{~K}$. 
the mode of electrical transport in Versicon. Moreover, this suggests that indeed the non-ohmic behavior of Version in PVC and the presence of the high field effect at low temperatures can be attributed to the presence of the PVC layers between the conducting Versicon aggregates. In addition, the data did not fit either of the ionic transport models confirming Versicon as an electronic polymer.

The data were thus fitted to a number of electronic transport models-conduction band, or hopping from localized state to conduction band which lead to resistivity given by $\rho=\rho_{o} \exp (E / k T)$, variable range hopping [13] between localized states near randomly distributed traps resulting in the conductivity given by $\sigma=\sigma_{o} \exp \left(-T_{o} / T\right)^{n}$ where $n$ may be $1 / 2,1 / 3$, and $1 / 4$, the Kivelson model [14] which involves phonon assisted hopping between localized states associated with randomly distributed dynamical defects and the conductivity is given by $\sigma=A T^{n}$, and the Fluctuation Induced Tunneling (FIT) model for composites consisting of spherical particles in which the transport is by tunneling with a potential barrier modulation by thermal fluctuations and the resistivity given by $\rho=\rho_{o} \exp \left(T_{1} /\left(T+T_{o}\right)\right)$.

The best fit was obtained for the FIT model (see Figure 5) as was obtained in Versicon in PVC [9], for all the samples studied. For the representative sample, the fit gave $T_{1}=417 \mathrm{~K}, T_{o}=94 \mathrm{~K}$ and $\rho_{o}=0.2133 \mathrm{ohm} \cdot \mathrm{cm}$. From the FIT model, the inter-particle distance is given by $s=\left[2 \delta\left(T_{1} / T_{o}\right)\right] / \pi$, where $\delta=\left(2 m_{e} V_{o} / \hbar^{2}\right)^{-1 / 2}$, and $V_{o}$ is the barrier potential, $m_{e}$ is the electron mass, and $\hbar$ is Planck's constant divided by $2 \pi$. The fit parameters yielded an inter-particle distance of $12 \AA$, using an estimated potential barrier of $0.2 \mathrm{eV}$ as was done for Versicon in PVC [9]. As can be seen in Figure 6, the high temperature data (above $120 \mathrm{~K}$ ) fits the thermal activation model very well, with an activation energy $E=0.015 \mathrm{eV}$.

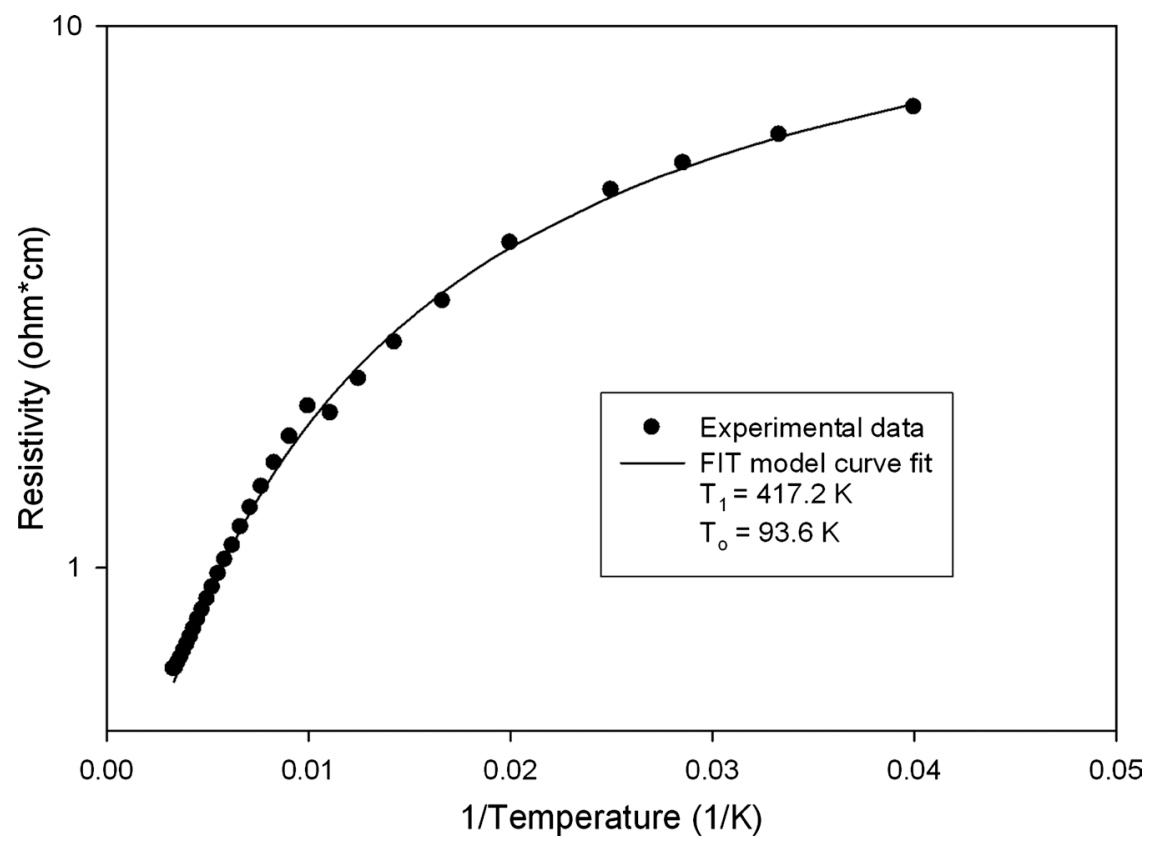

Figure 5. Log of resistivity versus $1 / \mathrm{T}$. The circles are measured data. The solid line is the fit to the Fluctuation Induced Tunneling model. 


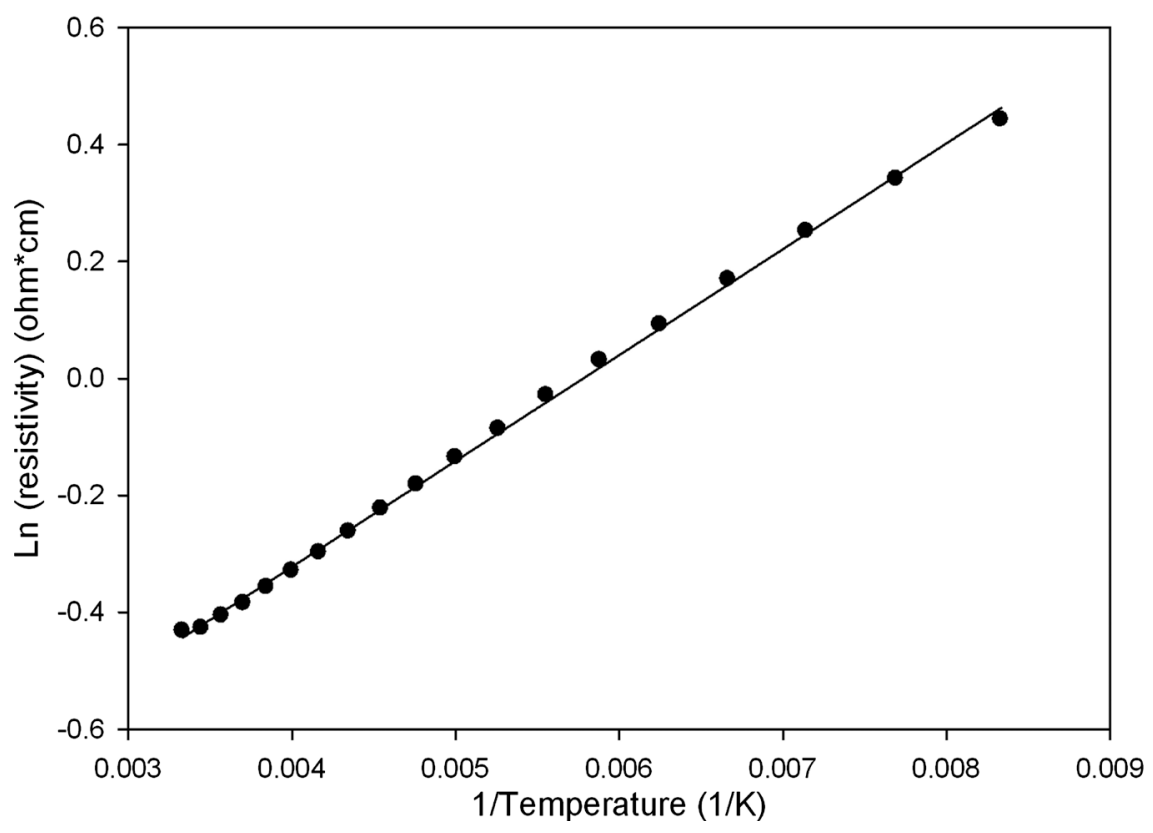

Figure 6. Log of resistivity versus $1 / \mathrm{T}$ for the high temperature data. The solid line is the fit to the thermal activation model.

The activation energy for hopping obtained $E=0.015 \mathrm{eV}$ in this work as compared to the $E=0.018 \mathrm{eV}$ obtained for the Versicon in PVC, as well as the estimated inter-particle distance of $12 \AA$ as compared to $13 \AA$, both suggest that Versicon has a strong tendency for aggregation even in an insulating matrix.

\section{Conclusions}

Versicon is stable and can be used for applications between temperatures of $-63^{\circ} \mathrm{C}$ and $163^{\circ} \mathrm{C}$. It forms conducting aggregates with inter-particle distance of $12 \AA$. This compares with $13 \AA$ obtained for Versicon in PVC [9] suggesting a strong tendency for aggregation to form conductive pathways in this material. Thermal activation as a means of electron transport is a parallel process to tunneling through the barrier, with the thermal fluctuations having the effect of lowering the activation energy [15]. As a result, at high enough temperatures, the thermal activation may dominate the mode of electrical transport in materials in which the FIT model is observed at low temperatures. Thus, as seen from Figure 6, thermal activation dominates the mode of electron transport in Versicon at high temperatures.

Other modes of electrical transport such as the variable range hopping model [9] [16] have been observed in polyanilines (PANI), suggesting that processing seems to affect the mode of electrical transport that eventually results in applications. It is thus imperative that the desired conductivity for specific applications be studied after the requisite processing. These results also suggest that Versicon, and other conducting polymers like it, can be easily incorporated in otherwise non conducting commercial paints to produce large scale conducting paint blends for various applications, with very little loss in conductivity. 


\section{Acknowledgements}

The author wishes to sincerely thank the following who contributed in various ways toward this project-former students Tom Angell and Fayez Assaf, Mr. Tom Chapman (Central Connecticut State University), Prof. James DeLaura (Central Connecticut State University), and Dr. C. C. Han (formerly of Allied Signal). Many thanks to the CCSU AAUP for providing grants to support this and other projects.

\section{Conflicts of Interest}

The author declares no conflicts of interest regarding the publication of this paper.

\section{References}

[1] Halford, B. (2007) A Polymer Interface for Prosthetics. Chemical \& Engineering News, 41.

[2] Lang, S.B. and Muensit, S. (2006) Lesser Known Piezoelectric and Pyroelectric Applications of Electroactive Polymers. Mater. Res. Soc. Symp. Proc., 889, 3.

[3] Raisanen, L. and Raissanen, H. (2006) Electro-Responsive Polymer and Its Sensor Applications. Mater. Res. Soc. Symp. Proc., 889, 45.

[4] Edelson, E. (1990) The Strangest Plastics. Popular Science, 90.

[5] Gupta, G. (2013) Development of Corrosion Resistant Conducting Polymers Coating for Aircraft Applications. PhD Thesis.

[6] Le, T.-H., Kim, Y. and Yoon, H. (2017) Electrical and Electrochemical Properties of Conducting Polymers. Polymers, 9, 150. https://doi.org/10.3390/polym9040150

[7] Stejskal, J. and Gilbert, R.G. (2009) Polyaniline. Preparation of a Conducting Polymer (IUPAC Technical Report). Pure and Applied Chemistry, 74, 857.

https://doi.org/10.1351/pac200274050857

[8] Shacklette, L.W., Han, C.C. and Luly, M.H. (1993) Polyaniline Blends in Thermoplastics. Synthetic Metals, 57, 3532-3537. https://doi.org/10.1016/0379-6779(93)90471-8

[9] Speel, R. and LeMaire, P.K. (1997) Direct Current Electrical Conductivity of Versi$\operatorname{con}^{\mathrm{Tm}}$ Blended in Poly(Vinyl Chloride). Journal of Materials Research, 12, 1183-1186. https://doi.org/10.1557/JMR.1997.0165

[10] Sauerbrunn, S. and Gill, P. Decomposition Kinetics Using TGA, TA Instruments (TA-075).

[11] Lukas, K. and LeMaire, P.K. (2009) Differential Scanning Calorimetry: Fundamental Overview. Resonance, 14, 808-817. https://doi.org/10.1007/s12045-009-0076-7

[12] Ratner, M. A. and Shriver, D.F. (1989) Polymer Ionics. MRS Bulletin, 14, 39-51. https://doi.org/10.1557/S0883769400061728

[13] Mott, N.F. (1987) Conduction in Non-Crystalline Materials. Clarendon Press, Oxford.

[14] Kivelson, S. (1982) Electron Hopping in a Soliton Band: Conduction in Lightly Doped $(\mathrm{CH})_{\mathrm{x}}$. Physical Review B, 25, 3798.

https://doi.org/10.1103/PhysRevB.25.3798 
[15] Sheng, P., Sichel, E.K. and Gittleman, J.I. (1978) Fluctuation-Induced Tunneling Conduction in Carbon-Polyvinylchloride Composites. Physical Review Letters, 40, 1197. https://doi.org/10.1103/PhysRevLett.40.1197

[16] Gmati, F., Fattoum, A., Bohli, N., Dhaoui, W. and Mohamed, A.B. (2007) Comparative Studies of the Structure, Morphology and Electrical Conductivity of Polyaniline Weakly Doped with Chlorocarboxylic Acids. Journal of Physics. Condensed Matter, 19, Article ID: 326203.

https://doi.org/10.1088/0953-8984/19/32/326203 